\title{
Laser-assisted chemical vapor deposition of 2D materials (Conference Presentation) (Withdrawal Notice)
}

Masoud Mahjouri-Samani

Masoud Mahjouri-Samani, "Laser-assisted chemical vapor deposition of 2D materials (Conference Presentation) (Withdrawal Notice)," Proc. SPIE 10907, Synthesis and Photonics of Nanoscale Materials XVI, 109070l (11 March 2019); doi: $10.1117 / 12.2509988$

SPIE. Event: SPIE LASE, 2019, San Francisco, California, United States 


\section{Laser-assisted chemical vapor deposition of 2D materials (Conference Presentation) (Withdrawal Notice)}

Masoud Mahjouri-Samani

Auburn Univ. (United States)

Proc. SPIE 10907, 109070I (2019)

Online Publication Date: 4 March 2019

Withdrawn from Publication: 11 March 2019

Conference Date: 2-3 February 2019

Conference Location: San Francisco, California, United States

Conference Title: Synthesis and Photonics of Nanoscale Materials XVI

Conference Chairs: Andrei V. Kabashin, Jan J. Dubowski, David B. Geohegan

Publisher's Note: This conference presentation, originally published on 4 March 2019, was withdrawn per author request. 